\title{
Erratum: Convexity of the Entanglement Entropy of SU(2N)-Symmetric Fermions with Attractive Interactions [Phys. Rev. Lett. 114, 050402 (2015)]
}

\author{
Joaquín E. Drut and William J. Porter
}

(Received 4 March 2015; published 1 April 2015)

DOI: 10.1103/PhysRevLett.114.139901

PACS numbers: 05.30.Fk, 03.65.Ud, 03.67.Mn, 67.85.-d, 99.10.Cd

We detected a sign error in our derivations, which were not fully included in the original version of our Letter. This leads to a modification of our original result. Here we present the corrected form of that result, which is of equally wide generality and applicability. The main connection established by our original work, namely, that a result derived in the context of nuclear physics has consequences for entanglement, remains valid.

Our original claim of the convexity property of the $n$th Rényi entanglement entropy $S_{n}$ can be written as

$$
S_{n}\left[\mathcal{B}^{j}, \mathcal{C}^{2 N-j}\right] \geq \frac{j-2 n_{1}}{2 n_{2}-2 n_{1}} S_{n}\left[\mathcal{B}^{2 n_{2}}, \mathcal{C}^{2 N-2 n_{2}}\right]+\frac{2 n_{2}-j}{2 n_{2}-2 n_{1}} S_{n}\left[\mathcal{B}^{2 n_{1}}, \mathcal{C}^{2 N-2 n_{1}}\right]
$$

An intermediate step in our derivations, omitted in the original version of our work, is actually a different convexity property which can be written as

$$
\mathcal{E}_{n}\left[\mathcal{B}^{j}, \mathcal{C}^{2 N-j}\right] \geq \frac{j-2 n_{1}}{2 n_{2}-2 n_{1}} \mathcal{E}_{n}\left[\mathcal{B}^{2 n_{2}}, \mathcal{C}^{2 N-2 n_{2}}\right]+\frac{2 n_{2}-j}{2 n_{2}-2 n_{1}} \mathcal{E}_{n}\left[\mathcal{B}^{2 n_{1}}, \mathcal{C}^{2 N-2 n_{1}}\right]
$$

where $\mathcal{E}_{n} \equiv \frac{n-1}{n} T S_{n}+F$. Here $T=1 / \beta$, where $\beta$ is the extent of the imaginary-time direction; $-\beta F=\ln \mathcal{Z}$, and $\mathcal{Z}$ is the partition sum (either in ground-state or finite-temperature approaches). Drawing a similarity with conventional thermodynamics, we will call $\mathcal{E}_{n}$ the entanglement energy. In ground-state methods, $F$ tends to the ground-state energy $E_{\mathrm{GS}}$ as $\beta \rightarrow \infty$. At that point, the entanglement energy coincides with $E_{\mathrm{GS}}$ as well. As shown by Lee [1], $E_{\mathrm{GS}}$ obeys a convexity property identical to that of Eq. (1). However that property does not allow the cancellation of the free-energy term in Eq. (2), and so both terms must be present in the definition of $\mathcal{E}_{n}$. Therefore, our proof only establishes Eq. (2) and not Eq. (1).

This work was supported in part by the U.S. National Science Foundation under Grant No. PHY1306520.

[1] D. Lee, Phys. Rev. Lett. 98, 182501 (2007). 